\title{
Impacto de los microcréditos en la reducción de la vulnerabilidad en la ciudad de Loja
}

\section{Impact of microcredits in the reduction of vulnerability in the city of Loja}

Lorena del Cisne Tacuri Peña

Universidad Internacional del Ecuador, Ecuador

Eduardo Aníbal Suarez

Universidad Internacional del Ecuador, Ecuador

Autor para correspondencia: lotacuripe@uide.edu.ec; edsuarezvi@uide.edu.ec

Fecha de recepción: 01 de Junio de 2017 - Fecha de aceptación: 15 de Agosto de 2017

Resumen: El presente estudio pretende desarrollar un análisis sobre la incidencia social de las microfinanzas en sectores vulnerables, bajo el contexto de las entidades financieras existentes en nuestro medio, y así analizar las perspectivas de impacto, tanto en el ámbito económico y su sostenibilidad financiera en el año 2016 como en el espacio social. Mediante un análisis comparativo con la aplicación de una investigación exploratoria y con la utilización de la técnica de la entrevista a las 16 cooperativas de ahorro y crédito que inciden directamente en dichos sectores y 349 encuestas determinando el resultado a través de la fórmula de la muestra y aplicada al segmento de sectores vulnerables mediante el método aleatorio; es así como la revisión de literatura ha generado indicios sobre la incidencia de las microfinanzas en los sectores vulnerables. El propósito del presente artículo es determinar cómo las instituciones microfinancieras experimentan una notable incidencia en el aspecto social, con la ampliación de su cobertura, rotabilidad constante del producto microcrédito y perfeccionando sus servicios, demostrando así su capacidad de adaptación y cambio a pesar de los diferentes fenómenos económicos y sociales, que se han visto afectadas en estos últimos años.

Palabras clave: financiero; incidencia; microfinanzas; social; sector vulnerable

\begin{abstract}
The present study aims to develop an analysis on the social impact of microfinance in vulnerable sectors, in the context of existing financial institutions in our country, and to analyze the prospects of impact, both in the economic area and its financial sustainability in the year 2016 as in the social space. By means of a comparative analysis with the application of an exploratory investigation and with the use of the technique of the interview to the 16 cooperatives of savings and credit that directly affect in these sectors and 349 surveys determining the result through the formula of the sample and Applied to the segment of vulnerable sectors by the random method; This is how the literature review has generated clues about the incidence of microfinance in vulnerable sectors. The purpose of this article is to determine how microfinance experiences a significant impact on the social aspect, with the expansion of its coverage, constant turnover of the microcredit product and perfecting its services, thus demonstrating its capacity for adaptation and change despite the different phenomena Economic and social problems that have been affected in recent years.
\end{abstract}

Key Words: Financial; Incidence; microfinance; social; vulnerable sector 


\section{Introducción}

Estos últimos años, algunas instituciones financieras, han enfocado sus colocaciones de crédito directamente al sector microempresarial, fundamentalmente en los sectores vulnerables manteniendo correspondencia con los objetivos tres y ocho del Buen Vivir y determinantes del comportamiento su dinámica y así poder identificar el impacto de la política pública, privada hacia este sector y formular recomendaciones para su mejoramiento. (Plan del Buen Vivir, 2013)

Actualmente en nuestra sociedad se observa que existen diversas comunidades que están desarrollándose y superándose mediante la implementación de nuevos negocios, pero se encuentran con grandes dificultades por la falta de recursos económicos, y el problema se agrava aún más, cuando no pueden acceder a mecanismos de financiamiento, debido a que las instituciones financieras no les prestan la facilidad de obtener créditos, puesto que como son personas con escasos recursos económicos, o forman parte de grupos vulnerables les es difícil cubrir las elevadas tasas de interés que les imponen dichas instituciones

\section{Problema}

La ciudad castellana del Sur de la Patria, enfrenta la crisis económica que invade a todo el país, lo que conlleva a que los diferentes sectores vulnerables la crisis incida más en su precaria economía.

El sector de las microfinanzas en la localidad juegan un papel muy importante en la economía local, lo que conlleva a que sus servicios de operación sean altos, de similar forma se evidencia que las tasas de interés para este servicio de microcréditos son reguladas por la Super Intendencia de Economía Popular y Solidaria.

Por tanto es fundamental resaltar que las instituciones microfinancieras, se han creado para contribuir a un desarrollo social, a través del financiamiento del producto del microcrédito, en beneficio de sus clientes, con tasas de interés y costos operativos-administrativos bajos.

Es así que la participación activa del sistema financiero en la colocación de estos fondos provoca cambios sociales a nivel del cliente, su familia y su entorno que le rodea, incidiendo en su bienestar social y económico.

- Las microfinanzas tienen altos costos de operación, altas tasas de interés para el microcrédito.

- Para un sector de la sociedad, para una economía deprimida.

- El objetivo del problema es que está dirigida o vinculada a los microemprendimientos.

- El producto es para que sea sostenible, para ello los prestatarios.

- Con ello tendremos la seguridad económica con el aumento neto de ingresos, acumulación de activos y la productividad laboral

\section{Revisión de literatura}


El presente estudio está enfocado a dar a conocer sobre los programas de financiamiento, que conceden las instituciones financieras, con su respectivo criterio social y solidario para sus clientes.

Con la participación de los actores financieros que intervienen en la prestación de los servicios de crédito, con los socios y personas directamente involucradas en el estudio, con el único fin de que sean corresponsables del desarrollo y de los sectores menos desprotegidos.

\section{Economía popular y solidaria y su sector financiero}

\section{Definición}

La Constitución del Ecuador indica en su artículo 283 que "el sistema económico es social y solidario" (Constitución, 2008). Asimismo especifica en su artículo 276 "El régimen de desarrollo tendrá los siguientes objetivos:

- Mejorar la calidad y esperanza de vida, y aumentar las capacidades y potencialidades de la población en el marco de los principios y derechos que establece la Constitución.

- Construir un sistema económico, justo, democrático, productivo, solidario y sostenible basado en la distribución igualitaria de los beneficios del desarrollo, de los medios de producción y en la generación de trabajo digno y estable.” (Constitución, 2008)

Al definir el sistema económico por su relación con la sociedad, por su valor central y por sentido antes que por determinada institucionalidad prefijada ideológicamente (como es el caso de neoliberalismo con respecto al mercado total), los constituyentes adoptaron una definición sustantiva y plural de economía, que no se define por los procedimientos de cálculo e instituciones de asignación óptima de recursos sino por garantizar de manera solidaria el sustento de todos los ciudadanos combinando diversas formas de organización económica.

“Art. 283. El sistema económico es social y solidario; reconoce al ser humano como sujeto y fin; propende a una relación dinámica y equilibrada entre sociedad, Estado y mercado, en armonía con la naturaleza; y tiene por objetivo garantizar la producción y reproducción de las condiciones materiales e inmateriales que posibiliten el buen vivir." (Constitución, 2008) 
La Economía Solidaria en la Economía Mixta

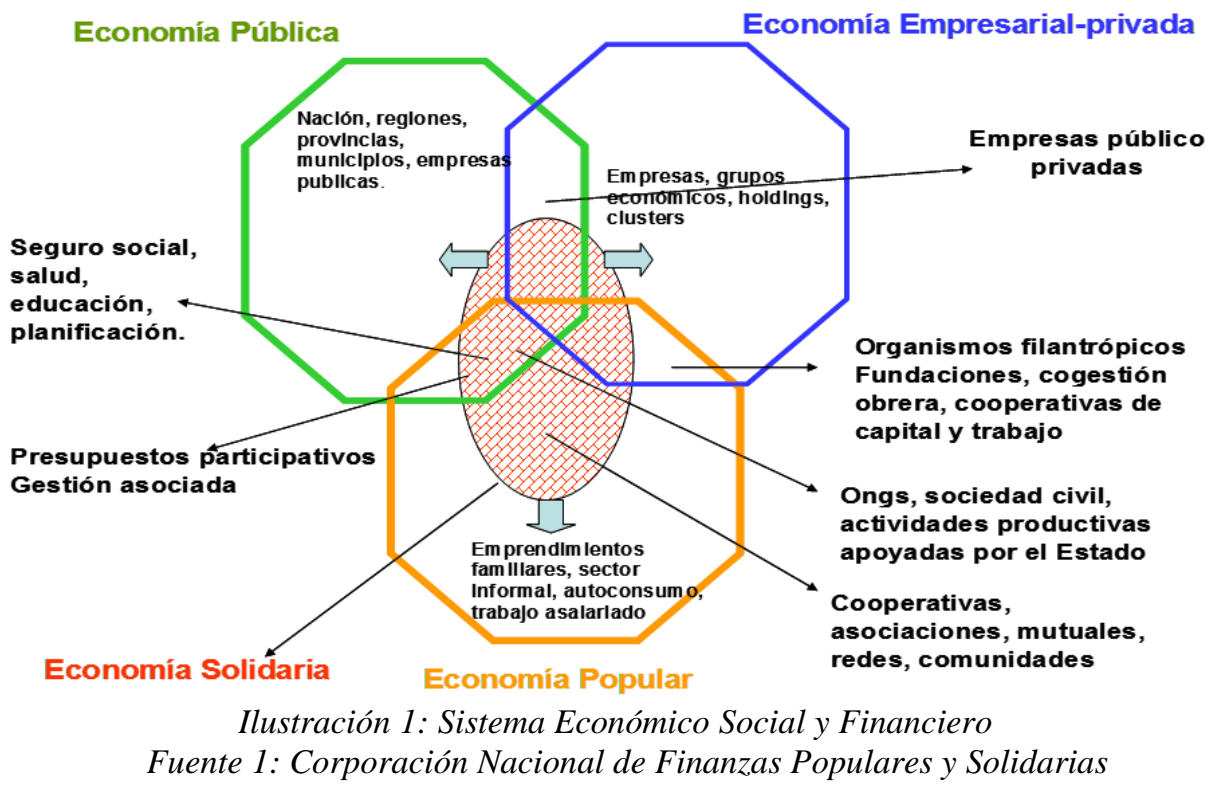

\section{Actores de las finanzas populares}

Según la Constitución de la República del Ecuador en su Art. 311 el sector financiero popular y solidario se compondrá de: "Cooperativas de ahorro y crédito, entidades asociativas o solidarias, cajas y bancos comunales, cajas de ahorro" (Constitución, 2008), reconociendo que todas estas formas sociales son de distinto tipo y tamaño y generalmente se han creado muchas de ellas en base a prácticas más equitativas y, son claves en el desarrollo social.

\section{El futuro de las microfinanzas.}

"Las instituciones micro financieras (IMF) afrontan importantes retos a futuro, que incluyen enfrentar las presiones que provienen del mundo político dado el importante caudal electoral que los usuarios de la IMF representan, y por otro lado también deben enfrentar los retos provenientes de la mayor competencia que supone una mayor participación de instituciones financieras tradicionales en este mercado" (Pineda \& Carvallo, 2010)

\section{Las microfinanzas y su impacto con la pobreza.}

Según Marulanda, "Las micro finanzas no son una herramienta de política para resolver los problemas de pobreza. Son, en esencia, un conjunto de productos financieros adecuados a clientes de bajos ingresos, que pueden contribuir a amortiguar las fluctuaciones de los ingresos y el flujo de caja de las personas, de modo que les permiten optimizar sus ingresos y desarrollar actividades productivas más rentables. Por esta vía, pueden ser un instrumento que contribuya a aliviar la situación económica de la población de bajos ingresos, sin que se puedan entender como un instrumento de política social." (Marulanda, 2007).

\section{Las instituciones de las microfinanzas.}


Las instituciones de microfinanzas han surgido como una repuesta para los microempresarios que requieren de un impulso para poder arrancar su negocio, el surgimiento de estas instituciones hace que se pueda tener acceso a recursos que le permitan incrementar su actividad productiva.

"Por lo tanto, las instituciones han orientado su estrategia a mejorar la gestión de riesgos (ventajosamente existe ya una norma para el sector de cooperativas de ahorro y crédito), a una revisión y actualización de su metodología crediticia e implementación de planes de gestión de cobranza. Se suma a esto, políticas de austeridad para el control de costos y gastos internos, ya que la afectación a los ingresos es evidente". (RFD, 2016)

\section{Cooperativas de Ahorro y Crédito}

Según la Ley de Cooperativas, señala que: "Son cooperativas las sociedades de derecho privado, formadas por personas naturales o jurídicas que, sin perseguir finalidades de lucro, tienen por objeto planificar y realizar actividades o trabajos de beneficio social o colectivo, a través de una empresa manejada en común y formada con la aportación económica, intelectual y moral de sus miembros" (Ley de Cooperativas, 2001)

\section{Microcrédito}

Unas de las generalidades del mercado financiero ecuatoriano ha sido la intensiva captación de depósitos dentro del sistema financiero nacional, lo cual representa una fuente importante de recursos económicos para financiar las operaciones del sector de las micro finanzas.

"Es todo crédito concedido a un prestatario, sea persona natural o jurídica, o a un grupo de prestatarios con garantía solidaria destinado a financiar actividades en pequeña escala de producción, comercialización o servicios, cuya fuente principal de pago la constituye el producto de las ventas o ingresos generados por dichas actividades, adecuadamente verificados por la institución del sistema financiero prestamista” (Junta Bancaría, 2002).

\section{Sectores Vulnerables}

En el Art.35 de la Constitución de la República del Ecuador considera a los sectores vulnerables como "personas adultas mayores, niñas, niños y adolescentes, mujeres embarazadas, personas con discapacidad" (Constitución, 2008)

-Servicios financieros diversificados dirigidos a los microemprendimientos.

-Sostenibilidad: desempeño económico y desempeño comercial, atravez de indicadores financieros como, Calidad de cartera, eficiencia y productividad, la gestión financiera, y la rentabilidad; hablar sobre la cartera de riesgo, microemprendimiento.

\section{Metodología}


La investigación realizada es de tipo documental ya que las informaciones obtenidas fueron de fuentes bibliográficas, además es una investigación descriptiva donde los datos obtenidos son tabulados y analizados, así mismo la investigación documental se caracteriza por el empleo predominante de registros, gráficos y más fuentes de información.

Es así como la presente investigación tienen como objeto realizar un estudio de las microfinanzas y su incidencia en los diferentes sectores vulnerables reconocidos.

- Diagnostico exploratorio del sector

- Encuesta del nivel de satisfacción de los prestatarios,

- Características socioeconómicas de los prestarios.

\section{Técnicas y procedimientos para recolectar la información.}

Para la recolección de la información se utilizaron técnicas y procedimientos sobre el tema a estudiar el mismo que consistió básicamente en la revisión de leyes relacionadas con las entidades que rotan y colocan más sus productos como es el caso de las cooperativas de ahorro y crédito específicamente con lo referente a microcréditos, libros, revistas, artículos relacionados con nuestro tema.

Una vez obtenida la información se procedió a realizar las diferentes tabulaciones y análisis de cada uno de los indicadores. Se elaboró un check list ajustado a los indicadores que genera el Banco Mundial en su portal online, el mismo que se le entregó a cada uno de los clientes de las 16 cooperativas de ahorro y crédito con mayor colocación del producto de microcrédito, y por ser un número total de 349 socios que pertenecen a sectores vulnerables información otorgada por las COAC, se aplica un censo para obtener una información más cercana a la realidad y beneficiosa para nuestro estudio, permitiéndonos así poder visualizar el criterio de cada socio, para realizar el análisis de incidencia se procedió a calcular de manera porcentual, conllevando así a conocer la incidencia de los microcréditos en los socios y su calidad de vida.

La estructura del referido Check list se lo notará en los resultados.

\section{Resultados}

Los resultados que se obtuvieron aplicando el Check y son los siguientes:

Tabla 1. Formato aplicado con resultados

\begin{tabular}{lcc}
\multicolumn{1}{c}{ Indicadores } & Si & Notal \\
\hline $\begin{array}{l}\text { Hijos Escolarizados, Y Familia Del Socio(A) Que Pagan } \\
\text { Todos L }\end{array}$ & $55 \%$ & $45 \%$ \\
Familia Del Socio(A) Que Han Accedido Al Servicio De & $86 \%$ & $14 \%$ \\
Salud & $73 \%$ & $100 \%$ \\
Familia Del Socio(A) Que Consumen Carne Al Menos & & $27 \%$ \\
Una Vez Al Mes & & $100 \%$ \\
\hline
\end{tabular}




\begin{tabular}{llll}
\hline $\begin{array}{l}\text { Número De Veces Que Han Comprado Ropa O Calzado } \\
\text { Durante Los Últimos Tres Meses }\end{array}$ & $81 \%$ & $19 \%$ & $100 \%$ \\
$\begin{array}{l}\text { Familia Del Socio(A) Que Han Realizado Mejoras En Su } \\
\text { Vivienda Durante Los Dos Últimos Años }\end{array}$ & $88 \%$ & $12 \%$ & $100 \%$ \\
$\begin{array}{l}\text { Familia Del Socio(A) Cuya Situación Económica Ha } \\
\text { Mejorado O Se Ha Mantenido En Estos Últimos 12 }\end{array}$ & $78 \%$ & $22 \%$ & $100 \%$ \\
Meses & &
\end{tabular}

Fuente 2: Chek List

De manera evidente los resultados demuestran que los indicadores base para el estudio como son educación, salud, alimentación, vestimenta y vivienda han mejorado en porcentuales satisfactorios superior al 50\% en todos los analizados; así mismo es relevante destacar el grado de mejoría que el socio(a) al acceder un microcrédito en estos 12 últimos meses existe la satisfacción de mejora y que equivale a un $78 \%$.

- Lista de microemprendimientos, a través del check list, con referencia a la calidad de vida y fuente trabajo mediante otras de variables de generación de información directamente a lo social.

- Trabajar el check list por sectores: los de menor incidencia en inversión por ejemplo: comida y bebida; Elaboración y venta de ropa, elaboración de bisutería y artesanías; y elaboración de productos de limpieza, servicio de cleanig (comprende lavado de ropa, carros, muebles, etc.) servicio de arreglo y alquiler de prendas de vestir, servicio de belleza.

\section{Discusión}

El porcentaje de hijos escolarizados entre las familias del socio(a), es del 55\% que tienen hijos en los diferentes establecimientos educativos de su zona y son capaces de cubrir los gastos escolares, frente al $45 \%$ de familias que tienen hijos escolarizados pero no pueden cubrir los gastos que demandan en los diferentes establecimientos; de acuerdo al resultado obtenido se determina que las familias que recibieron un microcrédito son capaces de cubrir los gastos en educación de todos sus hijos, siendo muy consecuentes en que la brecha de diferencia porcentual es muy reducida con una equivalencia de $10 \%$.

Con respecto a si el socio o familia han accedido al servicio de salud, el $86 \%$ han recurrido normalmente a los centros de salud estatales y privados; en tanto que el $14 \%$ no han concurrido, a pesar de ser significativa la diferencia porcentual y de acuerdo al análisis se pudo determinar que la mayoría de socios acceden a los servicios de salud regulados por el Estado y que en la actualidad el servicio es gratuito de acuerdo al Art. 362 de la Constitución de la República del Ecuador que en su pertinencia dice " La atención de salud como servicio público se prestará a través de las entidades estatales , privadas, autónomas, comunitarias y aquellas que ejerzan las medicinas ancestrales alternativas y complementarias . Los servicios públicos estatales de salud serán universales y gratuitos en todos los niveles de atención" (Constitución, 2008). 
Considerando el consumo de carne como un indicador de la mejora en la alimentación, en la tabla de resultados se puede observar que el $73 \%$ de las familias del socio(a), beneficiarias de un microcrédito consume carne al menos una vez al mes; frente al 27\%de familias de socio(a) que no consumen carne por su costo y falta de ingresos al hogar, enfatizando que según la FAO en su artículo titulado Crecimiento económico, hambre y malnutrición sostiene que "Aunque el crecimiento económico es importante para avanzar hacia la mejora de la nutrición de las personas, los vínculos son bidireccionales ". (FAO, 2012)

Las familias del socio(a) beneficiarios de un microcrédito tienen una mayor capacidad económica para hacer frente a determinados gastos como ropa, calzado entre otros, y han realizado compras significativas, lo que representa un porcentual del $81 \%$, frente al $19 \%$ de familias del socio(a) que no acceden a los microcréditos y no poseen los recursos suficientes para acceder a satisfacer la necesidad del indicador mencionado.

Las familias del socio(a) beneficiarios de un microcrédito tienen más posibilidades de realizar mejoras en su vivienda, un $88 \%$ contestan haber realizado mejoras en su vivienda durante los dos últimos años, frente a un $12 \%$ porcentual que no han realizado modificaciones en sus viviendas debido al coste de materiales y falta de ingresos a su hogar y que solo abastecen para cubrir gastos alimenticios y nada más.

Por último, cuando a las familias del socio(a) que se les aplico el check list se les pregunto sobre su situación económica durante los doce últimos meses, y como se observa en la tabla de resultados que el $78 \%$ corresponden al socio que ha optado por un microcrédito, manifiestan encontrarse mejor que el año anterior; en tanto que el $22 \%$ que corresponde a socios que no han accedido por un microcrédito en estos últimos meses, su situación económica no ha mejorado y se mantiene como el año anterior, lo que permite hacer referencia al criterio verbal del encargado del producto de microcréditos de una de las primeras instituciones solventes en la localidad como es la Cooperativa de Ahorro y Crédito Manuel Esteban Godoy. (Coopmego, 2013)

\section{Conclusiones}

De acuerdo a los indicadores aplicados, se determina que la población objeto de estudio que mediante los microcréditos se beneficia en el mejoramiento de su calidad de vida, haciendo más productiva sus actividades agrícolas, artesanales, negocios, etc., contribuyendo así al mejoramiento de la economía de su localidad.

Los sistemas de control para seguimiento del destino del microcrédito es de responsabilidad exclusiva del socio, por ende se debe considerar la implementación de un sistema de control sobre los destinos del microcrédito, con la única finalidad que tanto financieras como socia(a) puedan decidir sobre el destino de sus recursos, esto permitiría mayor transparencia en la relación cliente-financiera, promoviendo más participación en las actividades consagradas a viabilizar alternativas de desarrollo.

Como estándares de entrega y recepción de microcréditos, las entidades mantienen un riguroso proceso de selección para las captaciones y desembolsos del crédito, el agente de 
microcréditos es el encargado directo de realizar estas funciones con la finalidad de acelerar la circulación del dinero, captando así el excedente generado y hacerlo rotar la mayor cantidad posible logrando ampliar su capacidad multiplicadora de financiamiento y llegar así hacia los sectores vulnerables.

\section{Bibliografía}

Azofra, V., \& Femández, A. (1992). Evolución reciente de la modema teoría financiera. Anales de Ciencias Económicas y Empresariales , NQ7, 111-126.

Bernal, Cesar A. (2010). Metodologia de la Investigacion. In B. Cesar, Mettodologia de la Investigacion. Bogota: Orlando Fernandez.

Bernal, Cesar A. (2010). Metodologia de la Investigacion. In C. A. BERNAL, Metodologia de la investigacion (p. 111). Bogota: Orlando Fernandez.

Blank, L., \& Tarquin, A. (2002). Ingeniería Económica. México D.F.: McGraw Hill.

Buen Vivir. (S/D de S/M de 2013). Retrieved 17 de Mayo de 2016 from Buen vivir: http://www.buenvivir.gob.ec/objetivos-nacionales-para-el-buen-vivir

Business, D. (n.d.). www.doingbusiness.com.

CAN, C. A.

Cantarero. (2011).

Censos, I. N. (2016). Encuesta Nacional de empleo, desempleo y subempleo en el Ecuador.

CFMC. (2010). www.cfcm.com.

Constitución, 2. (2008). Constitución Dejemos el pasado atrás. Quito: Publicación oficial de la Asamblea Constituyente.

Coopmego. (17 de Septiembre de 2013). Megoline. From https://coopmego.com/megoline

Court Monteverde, E. (2012). Finanzas Corporativas. Buenos Aires: Cengage Learning.

Código Orgánico de la Producción, Comercio e Inversiones. Código Orgánico de la Producción, Comercio e Inversiones.

Damodaran, A. (2001). The Dark Side of Valuation. Prentice Hall.

Damodaran, A. (2005). Valuation Approaches and Metrics: A Survey of the Theory. Hanover: Foundations and Trends in Finance.

FAO. (S/D de S/M de 2012). Org. Retrieved 18 de Junio de 2016 from http://www.fao.org/docrep/017/i3027s/i3027s03.pdf 
Fernández, P. (noviembre de 2008). IESE Business School. Retrieved 30 de agosto de 2013 from Universidad de Navarra: http://ssrn.com/abstract=1266623

Fernández, P. (2008). Métodos de Valoración de Empresas. CIIF , 52.

Fernández, P. (1998). Valoración de Empresas. Barcelona: Gestión 200.

Gallegos Muñoz, C., \& Medina Giacomozzi, A. (2011). Determinación del valor económico añadido: un modelo alternativo. Contabilidad y Negocios , 18.

Gouge, O. d. (1791). Declaración de los derechos de la mujer y de la ciudadana.

INCOTERMS. (2010).

INEC. (2014).

Junta Bancaría. (2002). Resolución 457. 47. Quito: Junta Bancaría.

Ley de Cooperativas. (2001). registro Oicial nro. 400. Quito: Ley de Cooperativas.

López Lubian, F., \& De Luna Butz, W. (2002). Finanzas Corporativas en la práctica. Madrid : McGraw Hill.

Marr, B., Roos, G., Neely, A., Pike, S., \& Gupta, O. (2004). Hacia la tercera generación en la medición de resultados. Revista de Contabilidad y Dirección , 1, 31-46.

Marulanda, B. (2007). Microfinanzas y pobreza. Bogotá: Merpd- Departamento Nacional de planeación.

Milla Gutiérrez, A., \& Martínez Pedrós, D. (n.d.). Altair Consultores. Retrieved Agosto de 2013 from consultores.com/images/stories/publicaciones/Libro_Valoracion.pdf

http://www.altair-

Modigliani, F. y. (1958). The Cost of Capital, Corporation Finance and the Theory. American Economic Review , 261-297.

Nunes, M. B., Annansingh, F., \& Eaglestone, B. (2006). Knowledge management issues in knowledge-intensive SMEs. Journal of Documentation , 26 (I), 101-119.

Palenzuela, V. A., \& Herrero, G. d. (2008). La huella indeleble de Modigliani y Miller: MM. Boletín de estudios económicos 63 , 373-401.

Pereyra, T. M. (2008). Valoración de empresas: Una revisión de métodos actuales. Documento de trabajo No. 41, Facultad Administración y Ciencias Sociales, Universidad ORT Uruguay , 34 .

Pineda, R. \&., \& Carvallo, P. (2010). El futuro de la microfinanzas en América latina. Santiago de Chile: Cepal. 
Platón. Filósofo. Belleza y Estética. Gracia.

RFD. (17 de Mayo de 2016). www.rfd.org.ec. From www.rfd.org.ec: http://rfr.org.ec/index.php/blog/395-2016-08-17-17-16-13.html

Romero, L. Á. (2010). Principios de contabilidad. McGraw Hill.

Schumpeter. (1934).

SRI, S. d. (2014).

Stewart, G. (2000). En busca del valor. Barcelona: Gestión.

Universal, E. (martes de abril de 2013). El Universal. From El Universal: http://archivo.eluniversal.com.mx/notas/916992.html 